\title{
Valor diagnóstico de la fecha del coito fecundante para la estimación de la edad fetal
}

\author{
PAULINA LÓPEZ(1)
}

\section{RESUMEN}

Con el propósito de evaluar la fecha de coito fecundante para estimar la edad fetal, bajo la hipótesis de su alta capacidad predictiva, se estudió una cohorte de 243 mujeres embarazadas en control en un consultorio de atención primaria en la ciudad de Viña del Mar, en Chile. Cada embarazada tuvo tres determinaciones de la edad fetal: la primera a partir de las fechas coito fecundante y ultima menstruación; la segunda por ecografía precoz, y la tercera considerada como la edad de referencia, fue obtenida en el recién nacido mediante el examen de Ballard Extendido. Se realizaron análisis de correlación y concordancia entre las determinaciones y entre éstas y la edad de referencia; posteriormente se aplicó un modelo de regresión logística ordinal para establecer el nivel de precisión diagnóstica. Se observó que la edad fetal por dato coito fecundante se correlaciona estrechamente con la edad de referencia $(r=0,84), y$ más aún cuando la mujer presenta una fecha de ultima menstruación confiable $(r=0,90)$. Al análisis comparativo, el valor diagnóstico de la fecha estudiada no difiere significativamente con el de la ecografía precoz $(p=0,19)$. La ecuación de regresión logística alcanza una sensibilidad de $86 \%$ y una especificidad de $94 \%$ en presencia de una fecha de última menstruación confiable. Se concluye que la fecha del coito fecundante es un recurso semiológico útil para estimar la edad fetal, su precisión es comparable a la ecografia del primer trimestre del embarazo por lo que se sugiere su incorporación a la anamnesis obstétrica de rutina.

Palabras clave: Edad fetal, Ballard, coito fecundante, ecografía.

\section{ABSTRACT}

\section{DIAGNOSTIC VALUE OF THE FERTILIZABLE COITUS DATE FOR THE ESTIMATION OF FETAL AGE}

With the purpose of evaluating the date of the fecundating coitus for estimating fetal age, under the hypothesis of its high predictive capability, a cohort of 243 pregnant women being controlled at the primary care medical center of the city of Viña del Mar, in Chile, were studied. Each pregnant woman had three fetal age appraisals: the first, between the dates of fecundating coitus and last menstruation, the second, by means of precocious ecography, and the third, considered as the age of reference, was obtained from the newborn by means of the Extended Ballard examination. Correlation and concordance analyses between the determinations and between these and the frame of reference were performed; thereafter, a model of ordinal logistic regression

(1) Magíster en Bioestadística. Universidad de Valparaíso.paulinalopez@laposte.net 
was applied to establish the level of diagnostic precision. It was observed that the fetal age according to fecundating coitus is in close correlation with the age of reference $(r=0.84)$ and even more when the woman presents a date of the last reliable.menstruation $(r=0.90)$. Upon comparative analysis, the diagnostic value of the studied date does not significantly differ from that of the precocious ecography $(p=0.19)$. The logistic regression equation reaches a sensibility of $86 \%$ and a specificity of $94 \%$ in the presence of a reliable date of last menstruation. The conclusion is that the date of the fecundating coitus is a semiological resource useful for estimating fetal age; its precision is comparable to the first three-month ecography of pregnancy, for which reason its incorporation to routine obstetric anamnesis is recommended.

Key words: Fetal age, Ballard, Fecundating coitus, Ecography.

\section{INTRODUCCIÓN}

Determinar el momento del inicio de la gestación y con ello la edad fetal, ha sido desde los tiempos más remotos un gran desafío para la obstetricia y la pediatría. Esto porque se trata de uno de los diagnósticos más relevantes del período prenatal del cual derivan las conductas de vigilancia y cuidado del niño por nacer.

De los variados recursos con los que se cuenta actualmente para formular este diagnóstico, las fechas aportadas por la mujer gestante son las menos estudiadas. En efecto, en los últimos 20 años, las investigaciones para estimar con precisión la edad gestacional han apuntado a validar el uso de la ultrasonografía a través de patrones generales y regionales basados en la biometría embrionaria y fetal durante el primer trimestre de la gestación ${ }^{1-2}$.

Inicialmente se buscaron diámetros fetales con mayor capacidad predictiva, la mayoría de los estudios consideró entre sus referencias la fecha de última menstruación confiable ${ }^{3-5}$.

En la actualidad, se reconoce la ecografía como el recurso más preciso para determinar la edad fetal, por lo que muchos países la han adoptado como exploración sistemática del primer trimestre. Respecto a la fecha de la última menstruación, debe ser confirmada por la ecografía precoz, pero se sabe que no es válida en una alta proporción de mujeres (20\% a 45\%), ya que está sujeta a condicionantes externos y variaciones biológicas propias, además la naturaleza retrospectiva del dato puede adicionar sesgos a la calidad de la información 6-8.

En cuanto a la fecha del coito fecundante, desde los tiempos de la medicina romana se ha considerado como una referencia precisa para el cálculo de la edad fetal, pero los autores concuerdan en que esta fecha es muy difícil de obtener7-8. Este dato no se solicita sistemáticamente durante la anamnesis obstétrica; su valor diagnóstico es reconocido en la teoría y en la práctica, pero no ha sido estadísticamente probado.

Sin embargo, se puede constatar en la clínica obstétrica, la existencia de un grupo de mujeres que entrega con seguridad la fecha del encuentro sexual fecundante, la que es fácilmente reportada por la mujer como: "el día en que quedé embarazada".

Con base en lo anterior, se pretende evaluar la capacidad predictiva de esta fecha en términos de la precisión de la estimación de la edad gestacional fetal, a través de un análisis comparativo y de la aplicación de un modelo logístico 9 .

La hipótesis principal de esta investigación es que la fecha del coito fecundante permite obtener estimaciones muy precisas de la Edad Gestacional Fetal, considerada como tal aquella que no difiere en más de tres días con respecto a la edad fetal de referencia, y comparable a las estimaciones obtenidas por ecografía fetal del primer trimestre de la gestación.

Para ello se han planteado tres objetivos de estudio: el primero es determinar la precisión del diagnóstico de la edad fetal basado en la fecha del coito fecundante, teniendo por referencia la valoración de la edad gestacional en el recién nacido por examen clínico de Ballard Extendido. 
Posteriormente, comparar según 3 niveles ordinales de precisión, el diagnóstico de la edad fetal a partir de la fecha del coito fecundante frente al diagnóstico de edad gestacional fetal obtenido por ecografía sistemática precoz.

Finalmente, se pretende evaluar la capacidad predictiva de la fecha del coito fecundante según 3 niveles de precisión, en presencia y ausencia de una fecha de última menstruación confiable.

\section{MATERIAL Y MÉTODO}

Diseño de tipo observacional de evaluación de un medio diagnóstico, realizado en una cohorte de 250 embarazadas en control prenatal durante el periodo de junio 1998 a abril de 1999 , en el Consultorio Doctor Cienfuegos perteneciente al Servicio de Salud Viña Del Mar- Quillota en la ciudad de Viña del Mar, Chile.

Referencias de la población de origen de la muestra: En Chile, el 70\% de las mujeres asiste a control prenatal en los servicios públicos de salud. El Servicio de Salud Viña del Mar Quillota, concentra un vasto sector geográfico donde las mujeres beneficiarias de la atención provienen mayoritariamente de un medio urbano central y urbano periférico. El nivel socioeconómico más ampliamente representado es medio y medio bajo.

Muestra: Cohorte de 250 embarazadas en control en el Consultorio Doctor Cienfuegos. El tamaño obedece a dos criterios: corresponde al promedio de embarazadas en control durante el año 1997 (según Censo y estadísticas del servicio maternal),y al número esperado de mujeres en control prenatal en ese consultorio, según la estimación del Fondo Nacional de Salud al 30 de junio de 1998.

Unidad de análisis: Gestante que inició su control prenatal en el Consultorio Doctor Cienfuegos de Viña del Mar durante el período de recolección de datos (junio 1988 a abril 1999), y que tuvo su parto en el hospital Gustavo Fricke del Servicio de Salud Viña del MarQuillota.
Criterios de inclusión: embarazada en control prenatal, con una determinación de la edad gestacional por ecografía precoz (antes de las 12 semanas de gestación), cuyo parto tuvo lugar en el hospital Gustavo Fricke y que aceptó participar en la investigación.

Criterios de exclusión: Rechazo o imposibilidad por parte de la embarazada para responder a la información solicitada, asistencia a un único control prenatal, ausencia de examen ecográfico precoz, aquella mujer que no tuvo su parto en el hospital del servicio de salud.

\section{Criterios de selección del consultorio:}

a) socio-demográfico: El consultorio de Viña del Mar tiene características demográficas y socioeconómicas que corresponden a las de la población de embarazadas que se controla en el nivel de atención primaria en salud. La muestra incluye mayoritariamente los estratos con ingresos medios y bajos. Se observan mujeres en extrema pobreza provenientes de los sectores urbanos marginales.

b) Criterio clínico: El consultorio cuenta con disponibilidad de recurso médico especializado para la realización de la ecografía sistemática precoz, lo que entrega condiciones óptimas para esta determinación, el examen es realizado por dos médicos especialistas con el mismo ecógrafo, lo cual disminuye la variabilidad ligada al error de medición.

Por cada mujer gestante se realizaron tres mediciones de la edad gestacional fetal en el siguiente orden: la primera basada en la anamnesis a través de la fecha del coito fecundante (se consideró además la confiabilidad del dato fecha de la ultima menstruación: (F.U.M); la segunda mediante examen ecográfico sistemático antes de la semana 12 de gestación, y la tercera determinación se realizó en el post parto, mediante la valoración clínica de Ballard Extendido al recién nacido, y fue considerada para los fines de este estudio como la edad gestacional de referencia dado que es una de las evaluaciones clínicas más confiables ${ }^{10-11}$. 
Metodología de recolección de la información y control de sesgos de medición: Las determinaciones de la edad fetal fueron independientes entre sí con respecto al profesional que las formuló, al servicio en que se realizó y al momento del diagnóstico. Los datos y diagnósticos prenatales fueron recolectados en el consultorio por profesionales matronas a través de la ficha clínica, y de la aplicación de un cuestionario individual probado en 30 gestantes y corregido. Este fue aplicado en condiciones de privacidad, bajo consentimiento de la embarazada previa información de los objetivos del estudio.

Los datos correspondientes al diagnóstico neonatal de edad gestacional se obtuvieron desde la ficha de coordinación del recién nacido enviada desde el hospital al consultorio. Los datos faltantes fueron recogidos desde la ficha clínica en el hospital.

Trimestralmente se realizaron controles de calidad de la información recogida y del proceso de recolección de datos.

Se consideró que el examen de referencia puede presentar defectos de medición en los Recién Nacidos Pequeños para la Edad Gestacional $^{12}$, por lo cual se incluyeron en el plan de análisis pruebas suplementarias con el fin de determinar su influencia en las edades de referencia.

\section{Variables de estudio:}

a) Fecha del coito fecundante: Fecha única reportada por la mujer, coincidente con el período ovulatorio alrededor de la cual se produce la fecundación. A partir de este dato se establece el inicio de la gestación con una duración natural de 265 a 268 días hasta el término fisiológico ${ }^{13}$. Para fines de este estudio se ha calculado la fecha probable del parto adicionando 268 días a la referencia señalada por la mujer.

b) Ecografía precoz: Determinación de la edad fetal por ultrasonografía abdominal realizada por un médico especialista, desde la semana 6 hasta la semana 12 de gestación.

c) Fecha de última menstruación (F.U.M.): Fecha del primer día de la ultima menstruación reportada por la mujer al momento de ingresar al control prenatal. d) F.U.M. confiable: Fecha de la última menstruación reportada con seguridad por la mujer que presenta ciclos menstruales naturales y regulares en ausencia de factores endógenos o exógenos que puedan modificar la fisiología del ciclo menstrual.

e) F.U.M. no confiable: fecha de la última menstruación evocada por la mujer embarazada que no cumple con uno o más de los criterios de confiabilidad expuestos en el punto anterior.

f) Precisión del diagnóstico de edad fetal: Número de días de diferencia con respecto al diagnóstico de referencia.

g) Nivel de precisión de la estimación de la edad gestacional fetal: variable ordinal con tres niveles:

1: muy preciso: No más de 3 días de diferencia con la edad de referencia.

2: preciso: Entre 3 y 7 días de diferencia con la edad de referencia.

3: no preciso: Más de 7 días de diferencia con la edad de referencia.

Métodos estadísticos: la precisión de cada edad fetal se determinó por el Coeficiente de Correlación de Pearson.

El análisis comparativo entre las diferentes estimaciones de la edad fetal se realizó a través de la Dócima de Mac Nemar Extendida14. Posteriormente se determinó la concordancia entre diagnósticos a través del Coeficiente de Kappa ponderado. Finalmente se evaluó la capacidad predictiva de la fecha del coito fecundante a través de un modelo de regresión logística ordinal cuya variable respuesta fueron los 3 niveles de precisión definidos ${ }^{15}$.

Como apoyo técnico al procesamiento de los datos se utilizaron los programas estadísticos STATA versión 7.0 y SAS versión 8.1.

\section{RESULTADOS}

De la cohorte inicial se excluyeron 7 mujeres, la causa principal fue la pérdida durante el seguimiento hasta el momento del parto por cambio de domicilio. Ninguna mujer rechazó participar en el estudio y tres fueron excluidas por ausencia de ecografía precoz.

La cohorte estudiada fue de 243 embarazadas 
con las siguientes características: edad promedio de 25,3 años, escolaridad de 9,8 años, y un promedio de 1,2 gestaciones previas.

La fecha de coito fecundante fue entregada por 35 mujeres, lo que representa al $14,4 \%$ del total. En este grupo el 74,4\% presentó además una fecha de última menstruación confiable (Tabla 1).

Para el análisis descriptivo se determinaron los niveles de precisión para los diagnósticos de edad fetal por la fecha estudiada y por la ecografía precoz (Tablas 2 y 3 ).

La Tabla 4 muestra que la estimación de la edad fetal por la fecha del coito fecundante presenta una alta correlación con la edad fetal de referencia, esta correlación aumenta en presencia de F.U.M. confiable donde el valor del coeficiente de correlación de Pearson es de 0,90 .

El análisis comparativo por la dócima de Mac Nemar muestra que al interior de los tres niveles de precisión, no existen diferencias entre la edad fetal estimada por fecha del coito fecundante y por ecografía sistemática del primer trimestre $(\mathrm{p}=0,19)$.

El valor Coeficiente de Kappa Ponderado es estadísticamente significativo e indica una concordancia sustancial entre ambos diagnósticos ${ }^{16}$ cualquiera sea el nivel de precisión de la estimación de la edad fetal tanto en el grupo de mujeres con F.U.M. confiable como en el total (Tabla 5).

Con base en la alta correlación entre la edad gestacional fetal estimada por anamnesis y la edad gestacional real, se evaluó la capacidad predictiva de la fecha de coito fecundante a través

Tabla 1. Presencia del dato fecha del coito fecundante según confiabilidad de la fecha de la última menstruación (F.U.M). N=224

\begin{tabular}{ccrr}
\hline & Confiabilidad F.U.M. & Confiabilidad F.U.M. & Total \\
\hline Coito Fecundante & No & Si & \\
\hline NO & 89 & 100 & 189 \\
SI & 10 & 25 & 35 \\
Total & 99 & 125 & 224 \\
\hline
\end{tabular}

Tabla 2. Distribución del nivel de precisión de la estimación de la edad fetal por fecha del coito fecundante. $\mathrm{N}=35$

\begin{tabular}{cccc}
\hline Nivel Precisión & Frecuencia & $\%$ & Total \\
\hline Muy Preciso & 12 & 34,3 & 34,3 \\
Preciso & 11 & 31,4 & 65,7 \\
No Preciso & 12 & 34,4 & 100 \\
Total & 35 & 100 & 100 \\
\hline
\end{tabular}

Tabla 3. Distribución del nivel de precisión de la estimación de la edad fetal por ecografía precoz. N=240

\begin{tabular}{cccc}
\hline Nivel Precisión & Frecuencia & $\%$ & Total \\
\hline & 119 & 49,6 & 49,6 \\
Muy Preciso & 71 & 29,6 & 79,2 \\
Preciso & 50 & 20,1 & 100 \\
No Preciso & 240 & 100 & 100 \\
Total & & & \\
\hline
\end{tabular}


Tabla 4. Coeficiente de Correlación de Pearson entre las estimaciones y la referencia en el total de la muestra y en la muestra estratificada según los niveles de la variable confiabilidad del dato F.U.M. (*)

\begin{tabular}{ccc}
\hline & $\begin{array}{c}\mathrm{r} p \\
\text { total }(\mathrm{n}=35)\end{array}$ & $\begin{array}{c}\mathrm{r} p \\
\text { F.U.M. confiable }(\mathrm{n}=25)\end{array}$ \\
\hline Edad Fetal Por Coito Fecundante & 0.84 & 0.90 \\
Edad Fetal Por Ecografía & $\mathrm{P}<0.01$ & $\mathrm{P}<0.01$ \\
& 0.83 & 0.91 \\
& $\mathrm{P}<0.01$ & $\mathrm{P}<0.01$ \\
\hline
\end{tabular}

(*) La correlación con F.U.M. no confiable no se determinó $(\mathrm{n}=10)$

Tabla 5. Concordancia entre la edad fetal por ecografía precoz y fecha de coito fecundante.

\begin{tabular}{ccccc}
\hline & $\mathrm{N}$ & $\begin{array}{c}\text { Coeficiente } \\
\text { Kappa } \\
\text { Ponderado }\end{array}$ & $\mathrm{Z}$ & $\operatorname{Pr}>\mathrm{Z}$ \\
\hline FUM confiables y no confiables & 35 & 0.63 & 9.98 & 0.000 \\
FUM confiables & 25 & 0.66 & 7.47 & 0.000 \\
\hline
\end{tabular}

del siguiente modelo de regresión logística con respuesta ordinal:

Estimación No Precisa de la edad fetal = 22.69435+-.0835883* fecha del coito fecundante.

Estimación Muy Precisa de la edad fetal = $24.25818+-.0835883 *$ fecha del coito fecundante.

El ajuste del modelo fue evaluado en SAS a través la dócima de Wald y Chi cuadrado de Pearson. Aunque el tamaño muestral no es una exigencia rígida para la aplicación de la regresión logística, se acepta un tamaño muestral mínimo de 5 observaciones en cada uno de los niveles de precisión (Kock, G), condición que se cumple ampliamente en este estudio.

Las ecuaciones resultantes predicen los niveles Muy Preciso y No Preciso de la estimación de la edad fetal (Tabla 6). Para este último, la especificidad es de $73,9 \%$ y aumenta a $94,4 \%$ en presencia de una fecha de última menstruación confiable, igualmente el porcentaje de sujetos correctamente clasificados aumenta de $77 \%$ a $92 \%$. La sensibilidad de se mantiene alta en ambos casos. (Tablas 6 y 7).

El resultado confirma la capacidad predictiva de esta variable al obtener una muy buena combinación de sensibilidad y especificidad ${ }^{17}$ observada a través de la curva Roc. (Figura 1).

En cuanto a los análisis suplementarios; no hubo recién nacidos prematuros de muy bajo peso que afectaran la correlación con el Score de Ballard. Los Recién Nacidos Pequeños para la edad gestacional fueron 22 y este grupo no influyó en la determinación de la precisión.

\section{DISCUSIÓN}

Los resultados permiten definir la fecha del coito fecundante como un recurso semiológico de alta precisión, el conocimiento de su valor diagnóstico y su uso pueden ser de gran utilidad en la clínica obstétrica y perinatal.

En efecto, la ausencia de diferencia estadística significativa entre la estimación de la edad fetal por el dato fecha del coito fecundante y el examen ecográfico sistemático del primer trimestre, orienta hacia la elección de uno o de ambos recursos según el caso particular. Es decir, la presencia de la fecha de coito fecundante, acompañada de una F.U.M. confiable y un examen clínico normal, permite establecer con precisión la edad fetal y para tal efecto, puede reemplazar al examen ecográfico 
Tabla 6. Sensibilidad y especificidad del modelo de regresión logística ordinal para la predicción de la edad fetal a partir de la fecha de coito fecundante. $(\mathrm{N}=35)$

\begin{tabular}{|c|c|c|c|c|c|}
\hline Punto de Corte & Sensibilidad & Especificidad & VPP & VPPN & $\begin{array}{l}\text { Correctamente } \\
\text { clasificados }\end{array}$ \\
\hline $\begin{array}{c}\text { Estimación muy precisa } \\
\text { de la edad fetal por } \\
\text { c.f. }(0.34)\end{array}$ & $75 \%$ & $61.0 \%$ & $50.0 \%$ & $82.3 \%$ & $65.7 \%$ \\
\hline $\begin{array}{l}\text { Estimación precisa de la } \\
\text { edad fetal por c.f.(0.32) }\end{array}$ & $72.7 \%$ & $58.3 \%$ & $44.4 \%$ & $82.4 \%$ & $62.9 \%$ \\
\hline $\begin{array}{l}\text { Estimación no precisa de } \\
\text { la edad fetal por c.f.(0.34) }\end{array}$ & $83.3 \%$ & $73.9 \%$ & $62.5 \%$ & $89.5 \%$ & $77.1 \%$ \\
\hline
\end{tabular}

Tabla 7. Sensibilidad y especificidad del modelo de regresión logística ordinal para la predicción de la edad fetal a partir de la fecha de coito fecundante. en el grupo de mujeres con fecha de ultima menstruación confiable $(\mathrm{N}=25)$

\begin{tabular}{|c|c|c|c|c|c|}
\hline Punto de Corte & Sensibilidad & Especificidad & VPP & VPPN & $\begin{array}{l}\text { Correctamente } \\
\text { clasificados }\end{array}$ \\
\hline $\begin{array}{c}\text { Estimación muy precisa } \\
\text { de la edad fetal por } \\
\text { c.f. }(0.34)\end{array}$ & $77,8 \%$ & $62,5 \%$ & $53,8 \%$ & $83,3 \%$ & $68,0 \%$ \\
\hline $\begin{array}{l}\text { Estimación precisa de la } \\
\text { edad fetal por c.f.( }(0.36)\end{array}$ & $66,7 \%$ & $56,3 \%$ & $46,2 \%$ & $75,0 \%$ & $60,0 \%$ \\
\hline $\begin{array}{l}\text { Estimación no precisa de } \\
\text { la edad fetal por c.f.(0.28) }\end{array}$ & $85,7 \%$ & $94,4 \%$ & $85,7 \%$ & $94,4 \%$ & $92,0 \%$ \\
\hline
\end{tabular}

la edad fetal por c.f.(0.28)

sistemático del primer trimestre. Esto tiene relevancia en los lugares donde la ecografía de rutina no puede ser implementada, dado el alto costo económico o la carencia de recursos especializados.

A la luz de los resultados, este dato puede igualmente reemplazar una fecha de última menstruación no confiable y contribuir a resolver las dudas acerca de la edad fetal, que aún se plantean en grupos específicos de gestantes de alta vulnerabilidad psicosocial como lo son las adolescentes que inician tardíamente el control prenatal. Se puede plantear la hipótesis de que en el grupo de adolescentes sin vida de pareja estable, esta fecha sería más fácil de evocar por el carácter esporádico de los encuentros sexuales.

El hecho de que la precisión de la estimación de la edad fetal por la fecha del coito fecundante aumenta en presencia de una fecha de última menstruación confiable, da cuenta de la importancia que tiene la existencia de un grupo de embarazadas capaces de reunir ambas condiciones y, por lo tanto, aportar datos de calidad. De tal forma que si a nivel poblacional se aplica la probabilidad de obtener el dato, una de cada 7 mujeres que se controlan en el nivel primario de atención podría evocarlo, lo cual se traduce en un número importante. Considerando que el total de recién nacidos vivos fluctúa en Chile entre 250.000 y 300.000 , se puede estimar que 36.000 a 43.000 mujeres gestantes aportarían esta fecha en la consulta prenatal.

A diferencia de la fecha de la última 


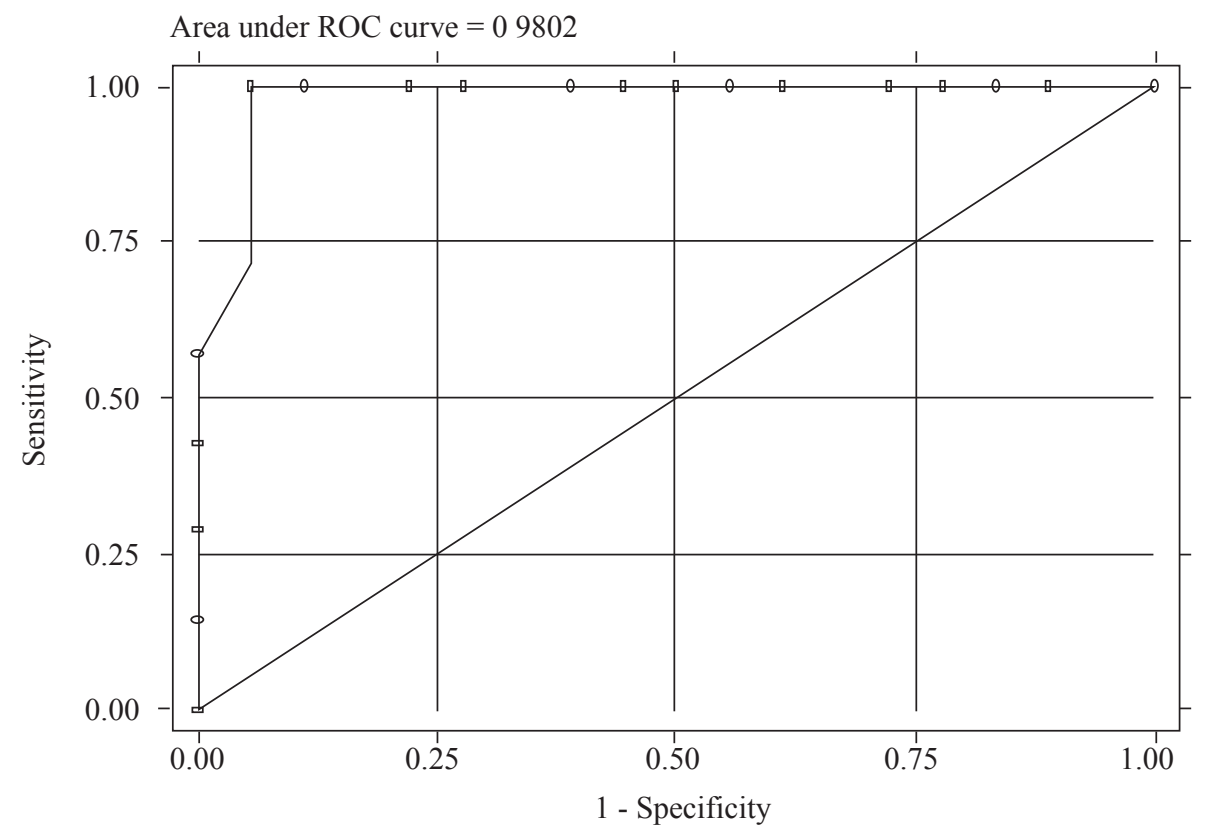

Figura 1. Curva Roc del modelo de regresión logística ordinal para la predicción de la edad fetal a partir de la fecha de coito fecundante en el grupo de mujeres con fecha de última menstruación confiable con $\mathrm{N}=25$. $(*)$

(*)La extensa área bajo la curva indica el alto poder discriminante de la ecuación de regresión logística que tiene como variable explicativa la fecha del coito fecundante y como variable respuesta el nivel de precisión de la estimación de la edad fetal.

menstruación, este dato no traduce un hecho fisiológico, se trata de un evento proveniente de la historia de la mujer y de la pareja, a ello se agrega la facilidad con la que puede ser recolectado desde el inicio al control prenatal.

Algunos autores señalan que el inicio de la gestación sería un momento propicio para recoger datos confiables porque los encuentros sexuales o "episodios genitales", son fácilmente evocados por la mujer en la primera consulta prenatal $^{19}$. En este sentido, la evocación de un encuentro sexual fecundante único plantea la posibilidad de estudiar la importancia subjetiva de este dato en la mujer y en el progenitor en términos de la significación afectiva que esta fecha podría tener en la historia prenatal y parental.

Cabe señalar que la ausencia de investigaciones previas acerca del valor diagnóstico de este dato recogido por anamnesis, no permite la comparación de resultados, de allí el interés que presenta validarlos también en otras poblaciones de mujeres embarazadas como lo es la población usuaria de los servicios privados de salud, que tiene características socioeconómicas diferentes, como también en la población de embarazadas provenientes de zonas rurales que no está representada en este estudio.

Los resultados acerca del valor predictivo de la fecha del coito fecundante, constituyen un aporte a la semiología obstétrica y, al mismo tiempo, confirman el valor asignado en la práctica ginecológica especialmente en los estudios de infertilidad, y en el ámbito médicolegal.

Considerando que aún cuando este dato no se puede obtener en la gran mayoría de las mujeres embarazadas, es de fácil acceso y no tiene costo económico, por lo que se sugiere incorporar durante la anamnesis obstétrica de rutina al momento del ingreso al control prenatal la pregunta formulada a las mujeres estudiadas: ¿conoce usted el día en que quedó embarazada?, con el fin de obtener y registrar en la ficha clínica esta importante referencia. 
Más allá de los beneficios objetivos de esta fecha, se puede suponer que al otorgar validez a lo que las embarazadas saben acerca de sus propios procesos reproductivos, no sólo se optimiza el enorme recurso que representa la anamnesis obstétrica, sino que, además, se le entrega mayor protagonismo y credibilidad. En el caso de la fecha del coito fecundante, a diferencia de la fecha de última menstruación, se agrega un reconocimiento implícito y precoz

\section{REFERENCIAS}

1.- LAGOS R, ESPINOZA R, ORELLANA J J. Curva de crecimiento fetal ¿buscando el estándar de oro? Rev. Chil. ultrason. 2002, 5 (3):83-89.

2.- MONGUELLI M, BISWAS A. A fetal growth estándar derived from multiple modalities. Early Hum Dev publicación en línea] 2001 jan, 60(3): 171-7. Se encuentra en: htpp://www.obgjmm@nus.edu.sg.

3.- SANTOS P, MACAHADO R, MOLINA J.R. Estudio de la discrepancia entre la edad gestacional referida (EGR) y la edad gestacional ajustada (EGA) en nuestro medio. Rev. Cuba. pediatr. 1985, 57(4): 428-441.

4.- DUTRA J, GILABERT M.I, COSTA E. Avaliacäo da idade gestacional: correlacäo entre data da última menstruacäo e ultra-sonografia. J. Bras. Ginecol. 1998, 108 (3): 55-57.

5.- GALVEZ J. Ecografía sistemática en el primer trimestre del embarazo. Rev. Chil. obstet. ginecol. 1993, 58(4): 323-327.

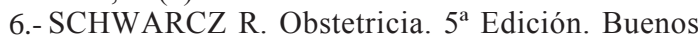
Aires: El Ateneo, 2003. p 117-134.

7.- MERGER R. Estudio clínico del embarazo normal en: Merger R, Levy J, Melchior J. « Compendio de Obstetricia » 6 ${ }^{\mathrm{a}}$ Edición. Paris: Masson, 2001. p 72-84.

8.- VACCARO H, OYARZÚN E. Estimación ultrasonográfica de la edad gestacional. En: Oyarzún E.,Gormaz G. Ultrasonografia en obstetricia. Santiago: Mediterráneo, 2003. p 29-40.
9.- LANDIS J, KOCH G. The Measurement Of Observer Agreement For Categorical Data. Biometrics 1977, 33: 159-174.

10.- TAPIA J L, VENTURA- JUNCA P. Manual de neonatología. Chile: Editorial Mediterráneo, 2000. p 65-70.

11.- CASTRO J, FUSTER P, LÓPEZ R, MENDEZ A, CORTABARRÍA C, DOMÉNECH E. Valoración de la Madurez del Recién Nacido. Hospital Universitario de Canarias. Servicio de Neonatología. Actas del Congreso de Pediatría. España: AEP, 2000. p. 1-7.

12.- MORAES C, REICHENHEIM M. Validación del Examen Clínico del Recién Nacido para la Estimación de la Edad Gestacional: Una Comparación entre el Score De Ballard Modificado con la fecha de la última menstruación y la ultrasonografía. Cuadernos de Salud Pública 2000, 16(1): 83-94.

13.- URANGA IMAZ F. Obstetricia práctica. Buenos Aires: Editorial Intermedica, 1977.

14.- AGRESTI A. Categorical Data Analysis, Ed Wiley series In probability and Mathematical Statistics, U.S.A, 1996. Pp. 347-373.

15.- HOSMER W, LEMESHOW S. Applied Logistic Regression, Ed A. Wiley inter science, U.S.A., 1989. Pp. 217-238.

16.- NORMAN G, STREINER D. Bioestadística. Madrid: Mosby, 1996. p 164-166.

17.- BLONDEL B, GOFFINET F, BRÉART G. Evaluation des soins en obstétrique. "Por una práctica basada en la evidencia» (fr.) Paris: Masson, 2001 p 24-52.

18.- RIEGELMAN R, HIRSH P. Como Estudiar un Estudio y Probar una Prueba. Organización Panamericana de la Salud. Washington DC: EUA: 1992. p 57-66.

19.- LANSAC J, BERGER C, MAGNIN G. «Obstetricia Clínica». $6^{\mathrm{TA}}$ ed. Paris: SIMEP, 2003. p48

20.- SHERWOOD RJ, MEINDL RS, ROBINSON HB, MAY RL. Fetal age: methods of estimation and effects of pathology. Departement of Anthropology University of Wisconsin U.S.A. [publicación en línea] 2000 nov, 113(3): 305-315. Se encuentra en:

htpp://www.rjsherwood@facstaff.wisc.edu.

Usted puede comentar éste y otros artículos publicados en la Revista Chilena de Salud Pública, enviando un correo electrónico a revistasp@med.uchile.cl 